\title{
Heterogeneity of disease-causing variants in the Swedish galactosemia population: Identification of 16 novel GALT variants
}

\author{
Annika Ohlsson \\ Karolinska Institute \\ Mary Hunt \\ Technological University Dublin, mary.hunt@tudublin.ie \\ Anna Wedell \\ Karolinska Institute
}

See next page for additional authors

Follow this and additional works at: https://arrow.tudublin.ie/scschbioart

Part of the Congenital, Hereditary, and Neonatal Diseases and Abnormalities Commons, Genetic Phenomena Commons, Genetic Processes Commons, and the Genetic Structures Commons

\section{Recommended Citation}

Ohlsson A, Hunt M, Wedell A, von Döbeln U. Heterogeneity of disease-causing variants in the Swedish galactosemia population: Identification of 16 novel GALT variants. J Inherit Metab Dis. 2019 Sep;42(5):1008-1018. doi: 10.1002/jimd.12136. Epub 2019 Aug 12. PMID: 31194895.

This Article is brought to you for free and open access by the School of Biological Sciences at ARROW@TU Dublin. It has been accepted for inclusion in Articles by an authorized administrator of ARROW@TU Dublin. For more information, please contact arrow.admin@tudublin.ie, aisling.coyne@tudublin.ie,gerard.connolly@tudublin.ie. Funder: Stockholm County Council; Karolinska Institute Research Foundation

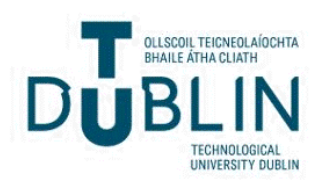


Authors

Annika Ohlsson, Mary Hunt, Anna Wedell, and Ulrika von Döbeln

This article is available at ARROW@TU Dublin: https://arrow.tudublin.ie/scschbioart/301 


\title{
Heterogeneity of disease-causing variants in the Swedish galactosemia population: Identification of 16 novel GALT variants
}

\author{
Annika Ohlsson ${ }^{1,2}$ (D) | Mary Hunt $^{3}$ | Anna Wedell ${ }^{1,4}$ | Ulrika von Döbeln ${ }^{1,2}$
}

${ }^{1}$ Centre for Inherited Metabolic Diseases (CMMS), Karolinska University Hospital, Stockholm, Sweden

${ }^{2}$ Department of Medical Biochemistry and Biophysics, Karolinska Institute, Stockholm, Sweden

${ }^{3}$ The School of Biological and Health Sciences, Dublin Institute of Technology, Dublin, Ireland

${ }^{4}$ Department of Molecular Medicine and Surgery, Karolinska Institute, Stockholm, Sweden

\section{Correspondence}

Annika Ohlsson, Centre for Inherited

Metabolic Diseases, L7:05, Karolinska

University Hospital, Stockholm SE-171

76, Sweden.

Email: annika.ohlsson@ki.se

Communicating Editor: Jaak Jaeken

Funding information

Karolinska Institutet, Grant/Award Number: KI-fonder; Stockholms Läns Landsting,

Grant/Award Number: ALF

\begin{abstract}
The aim was to determine disease-causing variants in the GALT gene which codes for the enzyme galactose-1-phosphate uridylyltransferase. Loss of activity of this enzyme causes classical galactosemia - a life threatening, treatable disorder, included in the Swedish newborn screening program since 1967. A total of 66 patients with the disease are known in Sweden and 56 index patients were investigated. An additional two patients with Duarte galactosemia were included. The disease-causing variants were identified in all patients. As reported from other countries only a few variants frequently recur in severe disease. The two variants p.(Gln188Arg) $(c .563 \mathrm{~A}>\mathrm{G})$ and $\mathrm{p}$. (Met142Lys) (c.425T $>\mathrm{A})$ are present in several index patients whereas the remaining are found in one to three patients each. The most common variant, p.(Gln188Arg), has an allele frequency of $51 \%$ in the cohort. A total of 16 novel variants were found among the 33 different variants in the cohort. Two of these are synonymous variants affecting splicing, demonstrating the importance of the evaluation of synonymous variants at the cDNA level. Concise sentence: Galactosemia is a rare disease in Sweden and the disease-causing variants are heterogenous including two synonymous variants.
\end{abstract}

\section{K E Y W O R D S}

galactosemia, genetics, mutations, neonatal screening, newborn screening

\section{1 | INTRODUCTION}

Galactosemia is a group of autosomal recessive disorders: galactokinase deficiency (OMIM \#230200), galactose-1-phosphate uridylyltransferase deficiency (OMIM \#230400, GALT deficiency), galactose epimerase deficiency (OMIM \#230350) and the recently discovered galactose mutarotase deficiency (GALM, OMIM \#137030) ${ }^{1}$ affecting the metabolism of galactose (Gal). The most common form is GALT deficiency resulting in an accumulation of Gal, galactose-1-phosphate (Gal-1-P), galactitol and galactonate. ${ }^{2}$

The disease is potentially lethal if not treated early with a Gal-restricted diet. Early diagnosis by newborn screening
(NBS) has improved the prognosis for patients. Long-term complications ranging from mild speech and language delay to more severe mental difficulties and neurological defects are not uncommon, and a high proportion of affected women have primary ovarian insufficiency. 3,4

Patients with a milder form of galactosemia are most often compound heterozygous for one severe, classical variant (G), and the milder, Duarte variant (D), p.(Asn314Asp) (c.940A>G). The p.(Asn314Asp) variant results in a residual activity of 50\% when it is found in cis with the four-base deletion, c.-119_116delGTCA in the promoter region, and the three intron variants c. $378-27 \mathrm{G}>\mathrm{C}$, c. $507+62 \mathrm{G}>\mathrm{A}$ and c. $508-24 \mathrm{G}>\mathrm{A} .^{5}$ This combination of cis Duarte variants is well known and is found in 
most studied populations. Patients with D/G are often detected in NBS programs due to a GALT activity of approximately $25 \%$ of normal. ${ }^{6}$ Although there is an uncertainty whether these patients need treatment, there are several publications indicating a favorable outcome for $\mathrm{D} / \mathrm{G}$ patients without treatment with a Gal-restricted diet. ${ }^{7-9}$

The first screening method described was a bacterial inhibition assay, ${ }^{10}$ followed by the Beutler enzyme spot test. ${ }^{11}$ The current methods employed by screening laboratories either measure the activity of the GALT enzyme (EC 2.7.7.12) and/or the amount of total Gal (Gal and Gal-1-P). ${ }^{12}$ Galactosemia screening, including all three enzyme deficiencies, was added to the Swedish NBS program in $1967 .^{13}$ The Beutler test was implemented in Sweden in 1986, and since then, the program only detects patients with GALT deficiency. The recall levels were adjusted in 1992 to minimize the detection of infants with the D/G variant. ${ }^{14}$

The Swedish NBS program is centralized to one laboratory, in Stockholm, with approximately 120000 babies screened annually. Centralization of the screening facilitates record retention of all recalled cases, true as well as false positive. The fact that all Swedish citizens have a unique personal identification number, minimizes the risk that patients are lost to follow up.

The screening laboratory is part of the Centre for Inherited Metabolic Diseases at Karolinska University Hospital, Stockholm, one of two Swedish laboratories performing biochemical and molecular diagnostics of inborn errors of metabolism. This includes molecular genetic investigations, determination of GALT activity in erythrocytes, analysis of Gal and Gal-1-P in dried blood spots (DBS) and galactitol in urine.

The first sequence variants, causing classical galactosemia, were reported in 1991. ${ }^{15,16}$ The most cited variants in Caucasians are p.(Gln188Arg) (c.563A $>$ G) and p.(Lys285Asn) (c.855G $>$ T), while p.(Ser135Leu) $(\mathrm{c} .404 \mathrm{C}>\mathrm{T})$ is common among patients of African origin. These three variants account for more than $80 \%$ of all affected alleles reported. ${ }^{17}$ Patients harboring the variant p.(Ser135Leu) have absent GALT activity in erythrocytes, but approximately $10 \%$ residual activity in tissues such as liver, intestine and leukocytes, resulting in a milder clinical picture. ${ }^{18}$

Here we present the spectrum of GALT variants in the Swedish galactosemia population, including 16 variants not reported to HGMD Professional 2018.3 (HGMD) or the ARUP GALT database, (http://www.arup.utah.edu/database/ galt/GALT_display.php, accessed 22 October 2018). ${ }^{19}$

\section{2 | MATERIAL AND METHODS}

\section{1 | Patients}

As at 31 December 2018, 5331800 newborns have been screened for galactosemia. Of these, 52 patients (29 males), have been diagnosed with severe GALT deficiency (GALT activity $<1 \%$ of normal) and five (two males) with mild GALT deficiency (GALT activity between $3 \%$ and $25 \%$ of normal and normal excretion of galactitol in urine from age 1 year). An additional nine patients (seven males) with classical galactosemia are known in the country: three patients diagnosed before the onset of screening and six in their home countries.

All galactosemia patients in Sweden, except one were screened for GALT variants in this or a previous study. ${ }^{20}$ The genetic study enrolled index cases from 56 families (32 males): age 1-54, median 25.8 years. Of these families, eight have more than one child with galactosemia. An additional two patients with Duarte galactosemia, are included.

The patients detected by NBS, were diagnosed at an average age of 8 days (range 3-14 days) before 2008, and at an average age of 4 days (range 3-6 days) from 2008, onwards. In 2008 the sampling time was moved from as soon as possible after 72 hours to as soon as possible after 48 hours of age. ${ }^{14}$

Genotyping is part of the clinical diagnostic routine for patients with galactosemia. Variants were confirmed in both parents of $60 \%(34 / 56)$ of the patients.

The project was approved by the Regional Ethical Committee of Stockholm (2008/351-31).

\section{I ANALYSIS OF THE GALT GENE}

\section{1 | DNA-sequencing}

Genomic DNA was isolated from whole blood with QIAamp DNA blood mini/midi kit (QIAGEN, Venlo, The Netherlands). Search for variants was performed by Sanger sequencing of the promoter, exons and intron-exon flanking regions in eight fragments. Out of the 11 exons, 3-4, 5-6 and 8-9 were sequenced together. Direct-cycle sequencing of all PCR fragments was performed with BigDye Terminator v3.1 Cycle Sequencing kit (Applied Biosystems, Foster City, CA) following the manufacturer's recommendations. Nomenclature for variants follows the guidelines and recommendations of the Human Genome Variation Society (http://www.HGVS.org/ varnomen $^{21}$ and novel variants were validated using the Mutalyzer program (https://mutalyzer.nl/). cDNA numbering commences from the ATG start codon, where +1 is the A of the ATG translation initiation codon (NM_00155.3). Novel variants have been submitted to ARUP GALT database.

\section{2 | Reverse transcription-PCR (RT-PCR)}

The influence of identified novel variants on mRNA splicing was studied by amplification of the segment containing the appropriate exons by RT-PCR. Total mRNA was extracted from whole blood using the PAXgene Blood RNA Kit (Qiagen GmbH, Hilden, Germany) or from fibroblasts. First 
strand cDNA was synthesized from isolated mRNA using iScript cDNA Synthesis Kit (Bio-Rad Laboratories Inc.). To confirm the aberrant splicing, cDNA fragments were amplified, using primers covering the area of interest. Amplified products were separated by agarose gel electrophoresis and visualized by gel red staining, GelRed, (Biotium Inc., Fremont, CA). Direct-cycle sequencing of the PCR fragments of interest, was performed with BigDye Terminator v3.1 Cycle Sequencing kit (Applied Biosystems, Foster City, CA).

\section{3 | Bioinformatic analysis}

The bioinformatic tools, Polymorphism Phenotyping v2 (PolyPhen-2 v2) ${ }^{22}$ and Protein Variation Effect Analyzer (Provean v1.1.3), ${ }^{23}$ were used for the prediction of the pathogenicity of unpublished nonsynonymous missense variants, and Berkeley Drosophila Genome Project (BDGP) ${ }^{24}$ was used for synonymous missense variants, splice site variants and intronic variants. Allele frequencies were obtained from Genome Aggregation Database (GnomAD), (http://gnomad. broadinstitute.org, accessed 15 May 2019). GALT variants were considered novel if not described in HGMD or the ARUP GALT database. All variants were checked against the Database of single nucleotide polymorphisms (dbSNP, https:// www.ncbi.nlm.nih.gov/snp, accessed 22 October 2018).

\section{4 | Biochemical analysis}

Gal and Gal-1-P were quantified in DBS. ${ }^{25}$ The detection limit at our laboratory was $0.1 \mathrm{mmol} / 1$ (reference value $<0.1 \mathrm{mmol} / \mathrm{l}$ ) for both analytes.

Galactitol was determined using GC-MS according to Allen with modifications. ${ }^{26}$ The detection limit was $20 \mu \mathrm{mol} / \mathrm{l}$ (reference value controls, $\mathrm{n}=24,<200 \mathrm{mmol} / \mathrm{mol}$ creatinine).

GALT activity was measured in erythrocytes with ${ }^{14} \mathrm{C}$ labeled Gal-1-P substrate ${ }^{27}$ after an incubation time of 1 hour (reference value controls, $\mathrm{n}=48,10.4 \mu \mathrm{kat} / \mathrm{kg} \mathrm{Hb}$, range 7.0-13.1 $\mu \mathrm{kat} / \mathrm{kg} \mathrm{Hb}$ ). In patients with possible residual activity, the GALT activity was measured with incubation times of 1 and 2 hours. To evaluate if prolonged incubation in the Beutler test could be an alternative for the determination of low GALT activity in erythrocytes, samples from patients were incubated for 3 and 6 hours in the Beutler test.

\section{4 | RESULTS}

\section{1 | Molecular analysis}

The search for GALT variants in the Swedish galactosemia population was initiated in the beginning of the 1990s. ${ }^{20}$ From a total of nine patients, five were fully characterized. These patients were homozygous for p.(Gln188Arg), as verified by restriction fragment length polymorphism. The remaining four patients were reinvestigated in this study.

Only data from index cases are included. In addition, two patients with Duarte galactosemia and novel variants are included. The disease-causing variants were detected in all patients and 33 different GALT variants were identified (Table 1).

Nineteen patients were homozygous and 17 heterozygous for the most common variant, p.(Gln188Arg). The second most frequent variant was p.(Met142Lys) (c.425T $>$ A), with an allele frequency of $9 \%$. Six patients were compound heterozygous for p.[(Met142Lys)];[(Gln188Arg)], making it the second most common genotype. Only three additional, known variants were found in more than one patient: $p$.(Leu195Pro) $(\mathrm{c} .584 \mathrm{~T}>\mathrm{C})(\mathrm{n}=2), \mathrm{p} .(\mathrm{Lys} 285 \mathrm{Asn})(\mathrm{n}=3), \mathrm{p} .(\operatorname{Arg} 333 \mathrm{Trp})$ $($ c. $997 \mathrm{C}>$ T) $(n=2)$ and c.508-29delT $(n=2)$.

A total of 16 novel variants were identified, each in only one patient, except for the two variants: p.(Leu37Arg) $($ c.110T $>$ G) and p.(Pro109=) (c.327A $>$ G), which were found in two patients (Table 1).

Nineteen patients carry genotypes not described before. Of these, 13 are associated with severe galactosemia, four with a milder disease and two are associated with the D/G disease (Table 2).

\section{2 | Prediction of pathogenicity of novel missense variants}

All nonsynonymous missense variants were analyzed with the bioinformatic tools PolyPhen-2 v2 and Provean (Table 1). Three novel variants were classified as benign in both tools. The in silico predictions for these variants are in agreement with the phenotypes of the patients. Four novel variants were considered deleterious by both tools and the clinical presentation of the patients supported these predictions.

For the novel variant p.(Ala46Val) $(\mathrm{c} .137 \mathrm{C}>\mathrm{T})$, the predictions were inconclusive. PolyPhen-2 considered the variant "Possibly damaging" while Provean considered it "Neutral." This variant was detected in a patient who is compound heterozygous for p.(Ala46Val)(;)(Asn314Asp) and diagnosed as D/G. The patient has a GALT activity of $25 \%$ of normal, and the p.(Ala46Val) is therefore considered a variant associated with severe galactosemia.

\section{3 | p.(Arg25Pro)}

The variant p.(Arg25Pro) (c.74G $>$ C) was found in combination with p.(Gln188Arg). The screening sample taken at age 5 days showed a GALT activity of 3\% in the Beutler test and slightly elevated Gal and Gal-1-P: 0.8 and $2.7 \mathrm{mmol} / \mathrm{l}$, respectively. At the time of recall (age 6 days) the patient did not present any clinical symptoms, despite Gal $3.9 \mathrm{mmol} / \mathrm{l}$ and Gal-1-P $2.3 \mathrm{mmol} / \mathrm{l}$. Urine galactitol at the 

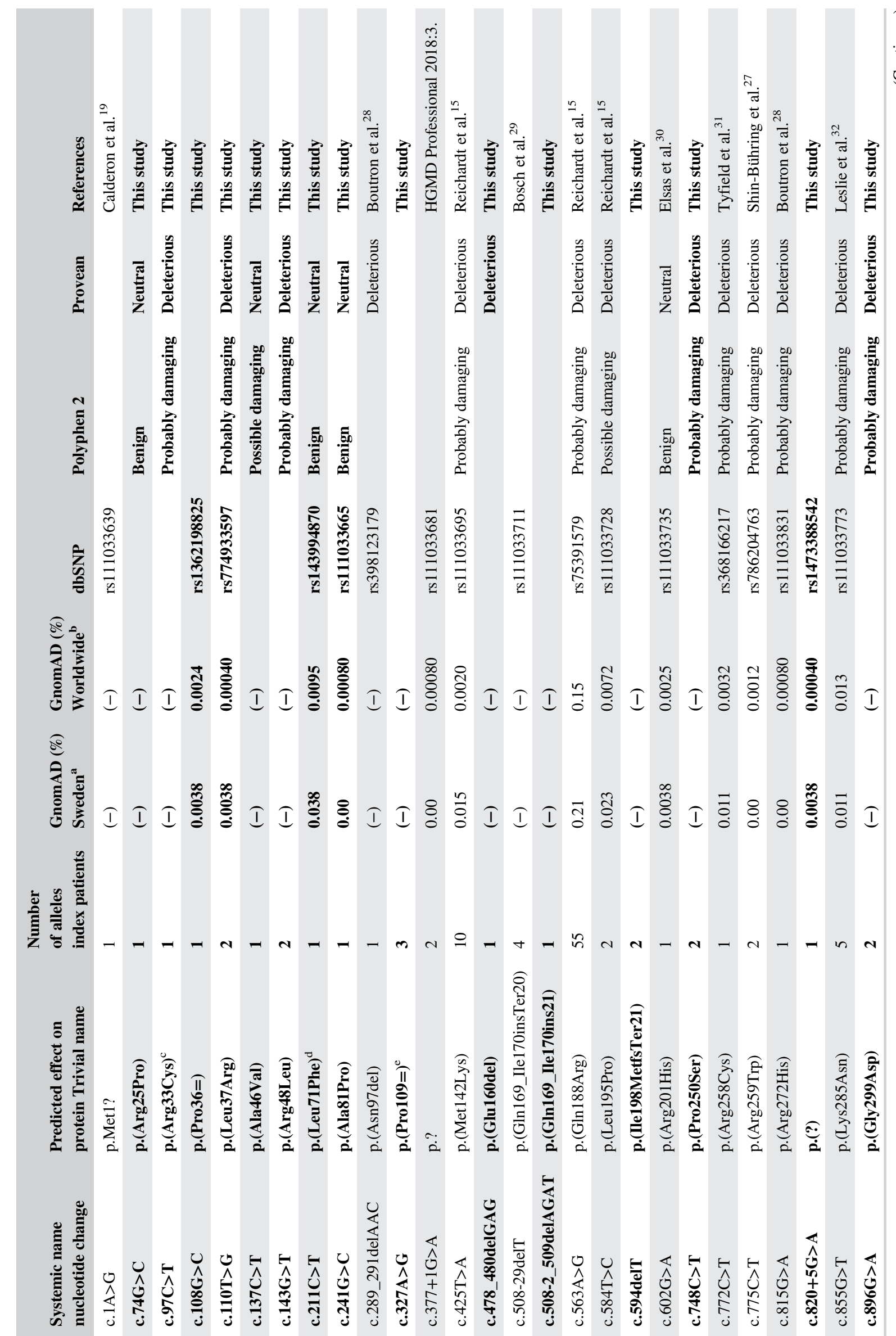


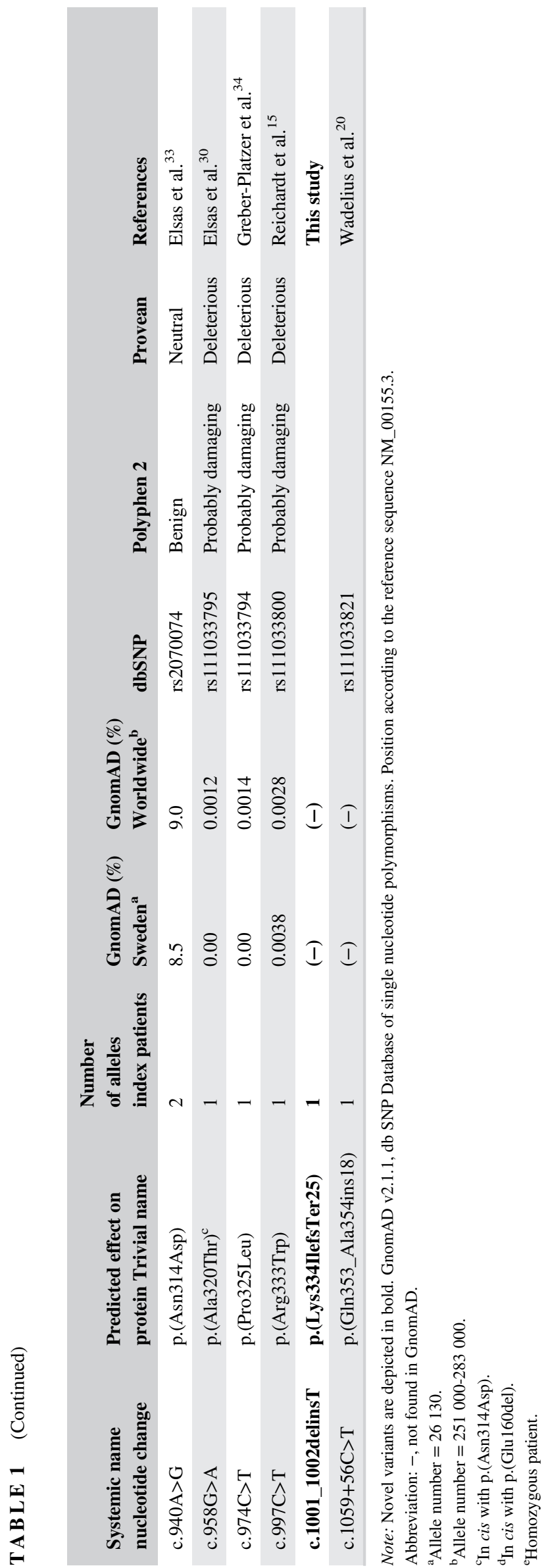

time of recall was $1940 \mathrm{mmol} / \mathrm{mol}$ creatinine, which is elevated but lower than usually seen in patients with classical galactosemia. Quantitative determination of GALT activity in erythrocytes with ${ }^{14} \mathrm{C}$-labeled Gal-1-P substrate showed an activity of $3 \%$ of normal (Table 3). The patient was classified as having a milder form of classical galactosemia and had normal secretion of galactitol in urine during dietary treatment.

\section{$4.4 \mid$ p. $($ Pro36=)}

The synonymous variant p.(Pro36=) (c.108G $>C)$ was detected in combination with $\mathrm{p}$.(Gln188Arg). The patient was re-called with a presumptive classical galactosemia. The patient had a residual activity of $9 \%$ in the Beutler test and the concentrations of Gal and Gal-1-P were 7.8 and $0.5 \mathrm{mmol} / \mathrm{l}$, respectively. Galactitol in urine was $9600 \mathrm{mmol} / \mathrm{mol}$ creatinine. The second DBS sample showed a GALT activity of $12 \%$ in the Beutler test. A prolonged incubation time in the Beutler test from 3 to 6 hours showed a proportional increase of fluorescence values over time. An increase in fluorescence is not seen in patients with two null variants (Figure 1A).

In silico analysis predicted the synonymous variant $\mathrm{p}$. (Pro36=) to create a new acceptor splice site 32 base pairs upstream of the authentic site and seven base pairs from the variant. The splicing pattern for $\mathrm{p}$.(Pro36=) was investigated in mRNA isolated from leukocytes. The cDNA transcript, when visualized on a $3 \%$ agarose gel after electrophoresis, showed two different bands-one of normal and one of shorter length (Figure 1B). Sanger sequencing of the shorter PCR product confirmed the in silico prediction. The $\mathrm{p}$. (Pro36=) variant is classified as mild and the patient had normal secretion of galactitol in urine during dietary treatment.

\section{5 | p.(Ala81Pro)}

The variant p.(Ala81Pro) (c.241G $>$ C) was found together with p.(Gln188Arg). The screening sample taken at age 3 days showed a GALT activity of $8 \%$ in the Beutler test and Gal and Gal-1-P levels were 0.3 and $1.9 \mathrm{mmol} / \mathrm{l}$, respectively. The same low activity in the Beutler test was found in the second DBS, with Gal and Gal-1-P levels of 0.6 and $1.4 \mathrm{mmol} / \mathrm{l}$. Galactitol in urine was $1050 \mathrm{mmol} / \mathrm{mol}$ creatinine. GALT activity in erythrocytes with ${ }^{14} \mathrm{C}$-labeled Gal$1-\mathrm{P}$ was $10 \%$ of normal. A longer incubation in the Beutler test from 3 to 6 hours showed a proportional increase of fluorescence over time, indicating that the patient has an ability to metabolize Gal-1-P to some extent, but at a slower rate than healthy controls (Figure 1A). The p.(Ala81Pro) variant is classified as mild and the patient had normal secretion of galactitol in urine without dietary treatment. 


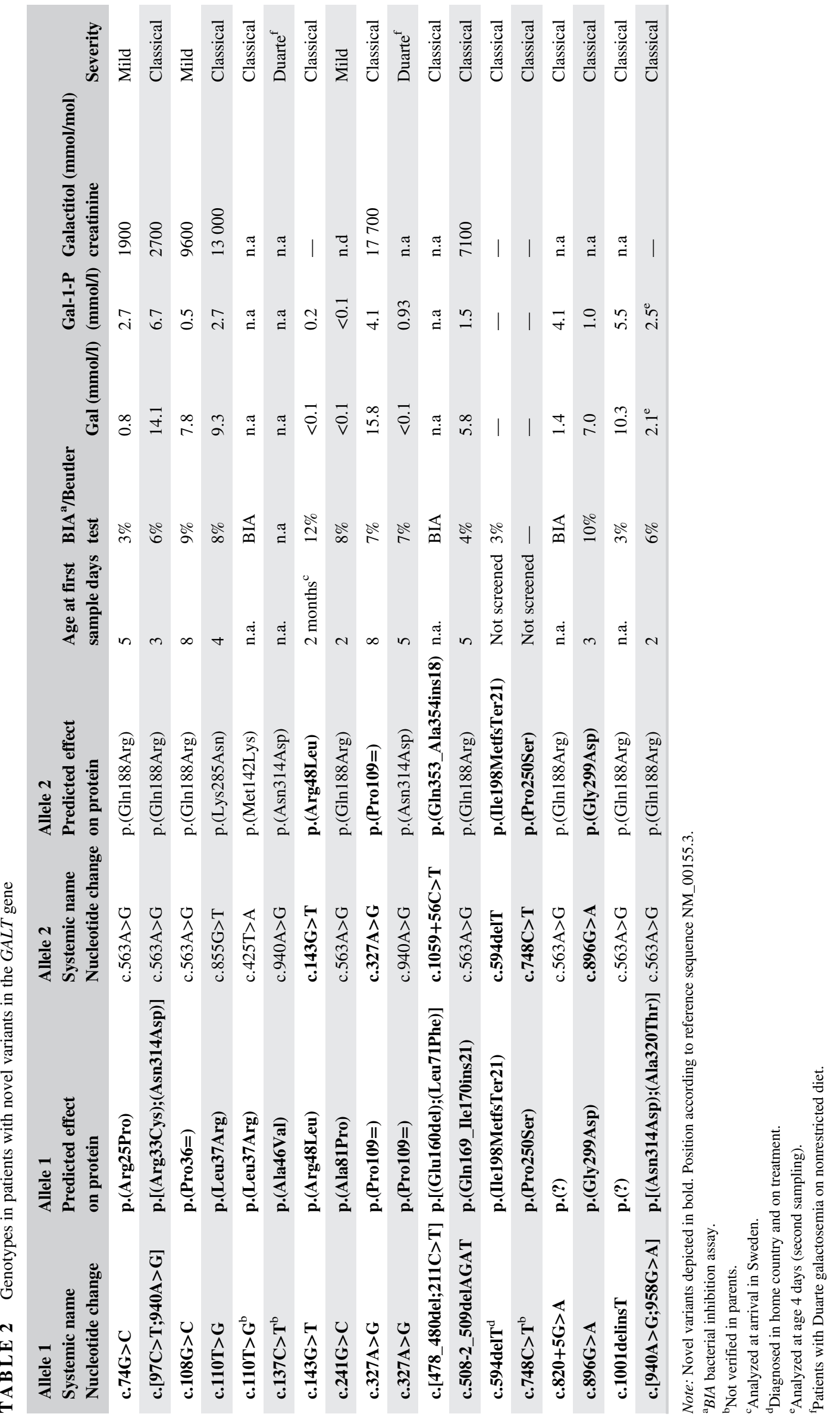




\section{6 | p.(Pro109=)}

The synonymous variant p.(Pro109=) $($ c.327A $>$ G), was found in a homozygous state in one patient with classical galactosemia and in compound heterozygous state together with p.(Asn314Asp) in a D/G patient.

The patient, homozygous for this variant, had a GALT activity of 7\% in the Beutler test and Gal and Gal-1-P values of 15.8 and $4.1 \mathrm{mmol} / \mathrm{l}$, respectively. Urine galactitol was markedly elevated, $17700 \mathrm{mmol} / \mathrm{mol}$ creatinine. Quantitative measurement of GALT activity in erythrocytes with ${ }^{14} \mathrm{C}$ labeled Gal-1-P showed an activity of $0.8 \%$ and $0.9 \%$ of normal after 1 and 2 hours of incubation, respectively (Table 3 ).

The second patient, who was compound heterozygous for p.[(Pro109=)];[(Asn314Asp)], also had a GALT activity of $7 \%$ in the Beutler test. The concentrations of Gal and Gal$1-\mathrm{P}$ were $<0.1$ and $0.93 \mathrm{mmol} / \mathrm{l}$, respectively. The patient

T A B L E 3 Erythrocyte GALT activity

\begin{tabular}{|c|c|c|}
\hline & 1 hour & 2 hour \\
\hline Genotype & $\begin{array}{l}\mu k a t / k g \\
\text { Hb (\%) }\end{array}$ & $\begin{array}{l}\mu k a t / k g \\
\text { Hb (\%) }\end{array}$ \\
\hline p. $[=] ;[=]$ & $9.8(100)$ & $18.0(100)$ \\
\hline p.[(Arg25Pro)];[(Gln188Arg)] & $0.26(3.0)$ & $0.47(2.6)$ \\
\hline p. $[($ Pro109=)];[(Pro109=)] & $0.08(0.8)$ & $0.17(0.9)$ \\
\hline p. $[(\operatorname{Gln} 188 \operatorname{Arg})] ;[(\operatorname{Arg} 333 \operatorname{Trp})]$ & $0.05(0.5)$ & $-0.05(0)$ \\
\hline
\end{tabular}

Note: GALT activity measured in erythrocytes at time point 1 and 2 hours in two patients with residual activity. has always been on an unrestricted diet and is classified as having D/G.

According to in silico analysis, the synonymous variant p.(Pro109=) is predicted to weaken the authentic donor site in exon 3, a decline in BDGP score from 0.90 to 0.36. The splicing pattern for p.(Pro109=) was investigated in mRNA isolated from fibroblasts. The cDNA transcript, when visualized on a 3\% agarose gel, showed four different bands which were subjected to Sanger sequencing (Figure 2A). The detection of a faint band of normal length probably explains
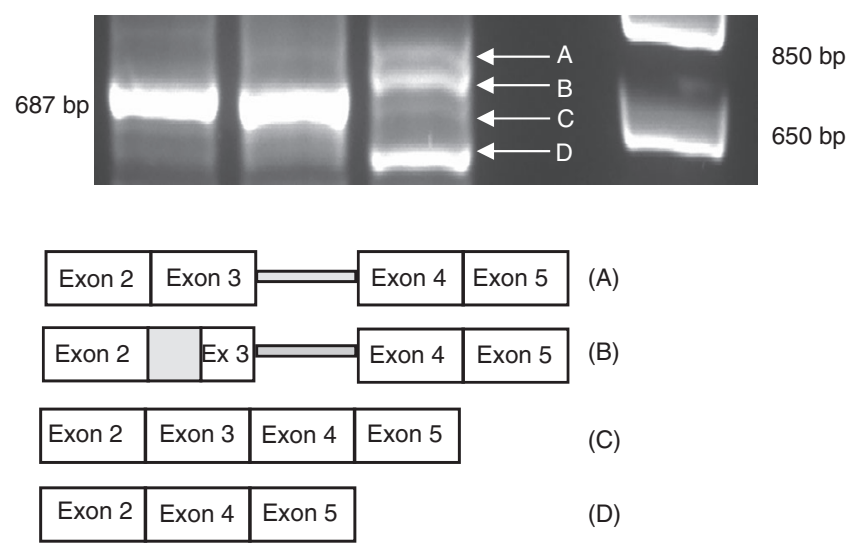

F I G URE 2 cDNA transcript after RT-PCR of the novel synonymous variant p.(Pro109=). The transcripts were visualized on a $3 \%$ agarose gel. Lane 1 and 2, wild type, lane 3 patient homozygous for p.(Pro109=), lane 4 size standard. (A) Insertion of intron

3, (B) skipping of 49 bp of exon 3 and insertion of intron 3, (C) wild type, (D) skipping of exon 3
(A)

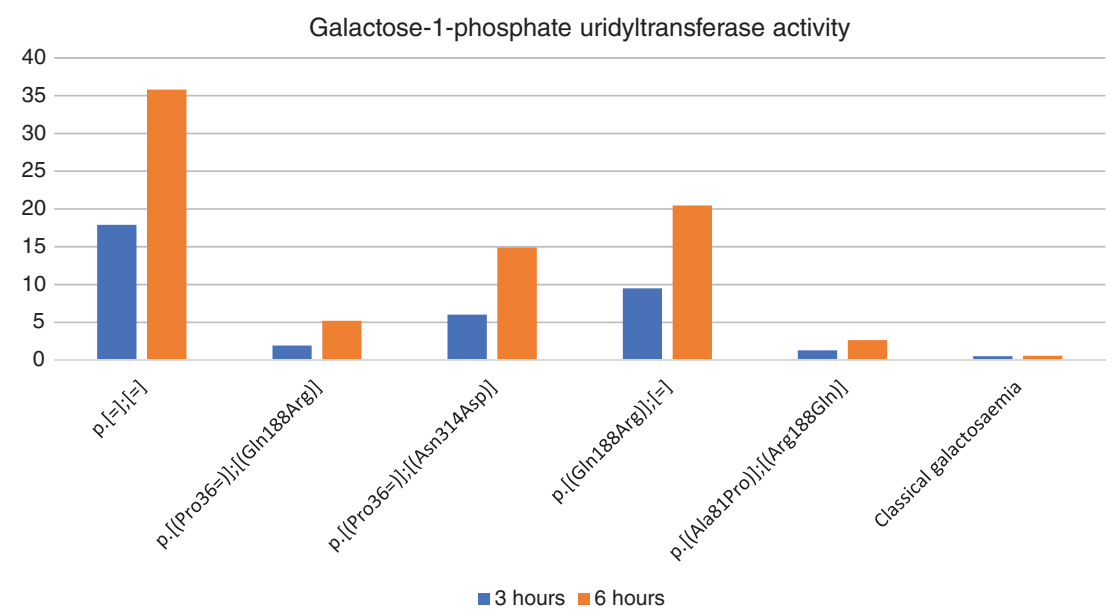

(B)

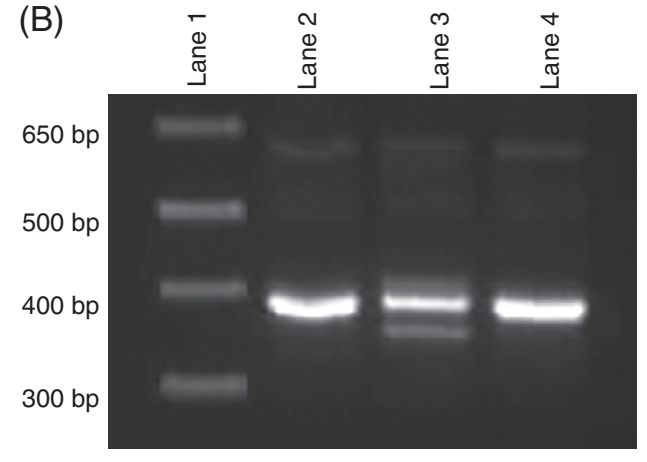

FIGURE 1 (A) Relative increase in fluorescence seen over time for the novel variants p.(Pro36=) and p.(Ala81Pro). Both these variants are in compound heterozygous state with p.(Gln188Arg) and analyzed with the Beutler assay. Fluorescence was measured at the time points 3 and 6 hours. Samples from a healthy individual, p. $[=] ;[=]$ and a patient who is compound heterozygous for p.[(Gln188Arg)];[=], 50\% GALT-activity, were used as controls. (B) cDNA transcript after RT-PCR of the novel synonymous variant p.(Pro36=). The transcripts were visualized on a $3 \%$ agarose gel resulting in an additional 32 bp shorter product. Lane 1, size standard, lane 2, patient compound heterozygous for p.[(Gln188Arg)];[=], lane 3 , patient compound heterozygous for p.[(Pro36=)]; [(Asn314Asp)], lane 4, control (wild type) 
T A B L E 4 Mendelian inheritance in a family with four $G A L T$ alleles

\begin{tabular}{llll} 
& Allele 1 & Allele 2 & GALT activity \% of normal in erythrocytes \\
Patient & p.[(Leu71Phe);(Glu160del)] & p.(Gln353_Ala354ins18) & 0.1 \\
Mother & p.[=] & p.(Gln353_Ala354ins18) & 50 \\
Father & p.(Leu71Phe) & p.(Asn314Asp) & 76 \\
Sibling & p.(Asn314Asp) & p.(Gln353_Ala354ins18) & 29 \\
\hline
\end{tabular}

the presence of a low residual activity of GALT in erythrocytes. The p.(Pro109=) variant is classified as a less severe variant of classical galactosemia.

\section{7 | p.(Glu160del)}

The novel in-frame variant, p.(Glu160del) (c.478_480delGAG), was found in $c i s$ with p.(Leu71Phe) (c.211C $>$ T). The patient was previously found to carry a cryptic splice site variant $\mathrm{p}$. (Gln353_Ala354ins18) $($ c.1059+56C $>\mathrm{T})$ in intron 10 on one allele. ${ }^{20}$ The cryptic splice site variant leads to an insertion of 18 amino acid as determined at the cDNA level. Mendelian inheritance estimation and GALT activity measurements were performed in the family (Table 4). The result of the measurements of GALT activity was inconclusive until sequencing analysis of the family was performed. Genetic analysis revealed, that the father was compound heterozygous for $\mathrm{p}$.(Leu71Phe) and p.(Asn314Asp), the latter being the Duarte variant. The sibling was compound heterozygous for the $\mathrm{D} / \mathrm{G}$ genotype, $\mathrm{p}$. [(Asn314Asp)];[(Gln353_Ala354ins18)]. The mother was carrier of the p.(Gln353_Ala354ins18). The p.(Glu160del) variant was not found in the father or mother indicating that it was a de novo mutation. According to the patient's phenotype, the in frame variant p.(Glu160del), is most probably associated with classical galactosemia.

Based on the father's genotype p.[(Leu71Phe)];[(Asn314Asp)] and GALT activity of $76 \%$ of normal in erythrocytes, the variant p.(Leu71Phe) is classified as benign. To our knowledge the variant p.(Leu71Phe) has not been described in patients with galactosemia (Table 1).

\section{8 $\mid$ c.508-29delT and c.508-2_509delAGAT}

The variant c.508-29delT has previously been described in a patient of Somali ancestry with classical galactosemia. ${ }^{29}$ The authors speculated that the variant may affect a branch point sequence in the GALT gene, resulting in a disruption of the authentic splice site. In our cohort, the variant c.508-29delT was found in a homozygous state in two patients of Somali ancestry as well.

The novel variant c.508-2_509delAGAT deletes the last two nucleotides in intron 5 and the two first nucleotides in exon 6 , compromising the authentic $3^{\prime}$ acceptor site. This variant was detected together with p.(Gln188Arg) in a patient with classical galactosemia.

In silico analysis with BGDP of each of these variants predicted the loss of the authentic 3 'acceptor splice (BDGP score 0.93), while creating a cryptic splice site (BGDP score of 0.98).

We investigated the aberrant splicing in mRNA from fibroblasts of a patient homozygous for c.508-29delT and from leukocytes of the patient heterozygous for the c.508-2_509delAGAT variant. Both variants activated the predicted cryptic splice site, between nucleotides -68 and -67 upstream of c.508 (Supporting Information, Figures S1 and S2).

\section{9 | Variants in cis with p.(Asn314Asp)}

Due to the high allele frequency of the Duarte variant, it is not uncommon to find the variant in cis with another galactosemia variant. In our study, the novel variant p.(Arg33Cys) (c.97C $>\mathrm{T})$ and the earlier described variant p.(Ala320Thr) $(\text { c. } 958 \mathrm{G}>\mathrm{A})^{30}$ were found in cis with p.(Asn314Asp). Both patients carry the common p.(Gln188Arg) variant on the second allele and have the classical phenotype.

\section{5 | DISCUSSION}

This is the first complete genetic study of patients with GALT deficiency in a Scandinavian country. All known patients, except one, are included in the survey. The birth incidence of GALT deficiency in Sweden is approximately $1 / 100000$, which is the same as in France and Italy, but lower than the reported incidence of 1/30 000-40000 for most other European countries. ${ }^{28,35,36}$

Thirty-three variants were detected in the Swedish galactosemia population, and of these 16 are unique for our cohort. Fifteen are not found in HGMD or ARUP GALT database and one has previously been published in $1993 .^{20}$ Only a few variants are frequent, which agrees with reports from other countries. ${ }^{30,31,37,38}$ The most common variant, among Swedish patients with classical galactosemia is p.(Gln188Arg) with an allele frequency of $51 \%$.

The missense variant, p.(Met142Lys), was one of the first variants identified to cause galactosemia. ${ }^{16}$ Although it is well described, this is the first time to our knowledge, that $\mathrm{p}$. (Met142Lys) is reported as the second most common variant 
in a population. Eight of the patients carrying this variant are of Swedish or Norwegian origin, while the ninth is from Lithuania.

Two novel variants were found in more than one patient, the variants p.(Leu37Arg) and p.(Pro109=). The two patients heterozygous for $\mathrm{p}$.(Leu37Arg) carry the variants $\mathrm{p}$. (Met142Lys) and p.(Lys285Asn) on the second allele, respectively. Both presented with severe clinical symptoms before the initiation of treatment.

The Duarte variant, p.(Asn314Asp), is found in all populations, albeit with different frequencies. According to the database GnomAD the highest allele frequency is found in South Asia (18\%), as compared to an allele frequency of 9.4\% in Europe (non-Finnish). The lowest frequency is found in East Asia (0.9\%). At least nine missense variants and four nonsense variants have previously been described in cis with the Duarte variant (Supplemental Table S3). ${ }^{28,30,31,39-42}$

The two complex variants p.(Arg33Cys;Asn314Asp) and $\mathrm{p}$. (Asn314Asp;Ala320Thr) in our population increase this group to 15 . These results demonstrate the importance of sequencing the whole $G A L T$ gene and not only a panel including common variants. If only a panel is analyzed and p.(Asn314Asp) is included, there is a risk of misdiagnosis, as the p.(Asn314Asp) variant frequently occurs in cis with more rare variants.

Occasionally, patients with $D / G$ are recalled because of a GALT activity $<15 \%$ in the Beutler test. The patient who is compound heterozygous for p.[(Ala81Pro)];[(Gln188Arg)] was recalled due to a residual activity of $8 \%$, which was the same in the repeat sample, while Gal-1-P was only slightly elevated. Genotyping detected the novel variant p. (Ala81Pro). A disease-causing variant in the same codon $\mathrm{p}$. (Ala81Thr) (c.241G >A) has been described..$^{32}$ To assist estimation of the pathogenicity of p.(Ala81Pro), the variant was evaluated in PolyPhen-2, with the result "Benign," in contrast to p.(Ala81Thr), which was denoted "Probably damaging," The variant p.(Ala81Pro) reduces GALT activity in the Beutler test to the same extent as in classical galactosemia patients. Blood samples from the patient with this mutation in combination with p.(Gln188Arg) still have the ability to metabolize Gal-1-P, although at a slower rate than samples from healthy controls. This was seen when measuring GALT activity quantitatively in erythrocytes as well as in a prolonged Beutler test. We consequently estimate that the variant p.(Ala81Pro) results in a severity equal to Duarte galactosemia. The patient has been on a normal diet from 1 year of age, is close to 10 years old and has had a normal development. All the patients with mild galactosemia have had a normal secretion of galactitol after the newborn period.

The patient with the de novo mutation described here illustrates the importance of sequencing the complete $G A L T$ gene in all family members when the results of the measurement of GALT activity are inconclusive.

\subsection{Impact of synonymous variants}

Synonymous variants in the GALT gene are common. The ARUP GALT database and Exome Aggregation Consortium (ExAC) (http://exac.broadinstitute.org, accessed 22 October 2018) contain 12 and 44 unique synonymous variants, respectively. An additional seven variants are found in both databases. Fifteen of the synonymous variants in the ARUP GALT database are described as benign, whereas four are of uncertain severity. After in-silico analysis of all 63 variants in BDGP, only six variants were predicted to either abolish or weaken an existing splice site or create a new splice site. Included in the six synonymous variants, predicted to affect splicing, are the three variants proven to be pathogenic: $p$. (Pro36=), p.(Pro109=) and the earlier presumably benign synonymous variant, p. $(\mathrm{Gly} 338=)(\mathrm{c} .1014 \mathrm{C}>\mathrm{G})$. The latter has been shown to be disease-causing by activating an alternative splice donor site, resulting in the exclusion of 46 nucleotides at the end of exon $10{ }^{28}$ The two synonymous variants described in this article both result in reduced GALT activity. The variant p.(Pro36=) activates a new acceptor splice site, excluding 32 nucleotides at the beginning of exon 2, giving a shorter protein. Amplification of GALT cDNA carrying the p.(Pro109=) variant revealed the presence of multiple fragments from RT-PCR (Figure 2A, B). This strongly suggests that the p.(Pro109=) allele in this patient generates a normal transcript, two longer and one shorter transcript, supporting the idea that all four 5 'splicing sites, cryptic and normal are used.

Splice variants that generate both normal and abnormal transcripts from the same allele are referred to as leaky. The relative amounts of normal and aberrant splice products generated are important; the higher the levels of normal transcript, the milder the phenotype. The presence of some normal transcript is probably generating the residual GALT activity of approximately $1 \%$, which is supported by the 1- and 2-hour incubation of erythrocytes with ${ }^{14} \mathrm{C}$ labeled Gal-1-P and the somewhat milder phenotype of the patient. Our results, together with the results of Bourton et al 2012, strengthen the importance to evaluate the consequences of synonymous variants at the mRNA level, especially if no other variants in the GALT gene are detected. ${ }^{28}$

Classical galactosemia is a potentially progressive disease. ${ }^{4}$ Our experience from the three patients described in this paper with some residual activity is that a GALT activity of $1 \%$ is not enough to prevent severe symptoms before diagnosis or to prevent long term complications when the patient is on Gal restricted diet.

Sweden moved the sampling time from as early as possible after 72 hours, to as early as possible after 48 hours of age in 2008. An important advantage of the earlier sampling time is that patients are recalled at a younger age. This 
increases the chance of diagnosis and commencement of treatment, before the development of life-threatening symptoms.

\section{6 | CONCLUSIONS}

Classical galactosemia is a rare disease in Sweden affecting 1/100 000 newborn infants. Like in other countries only a few variants in the GALT gene are frequent in the cohort. In this study 16 of the 33 variants are novel including a probable de novo variant.

The variants p.(Pro36=) and p.(Pro109=) are two new synonymous variants in the GALT gene proven to be pathogenic. This demonstrates the importance of evaluating the consequences of synonymous variants in the GALT gene at the cDNA level.

\section{ACKNOWLEDGMENTS}

We are grateful to Anna Malmberg, Birgitta Öberg, Marianne Söderkvist, Britt-Marie Ruuth, Claes Guthenberg, Maire Kotajärvi, Ingrid Westman, Helene Bruhn and Nicole Lesko for sharing their knowledge with us about NBS and different DNA-techniques.

\section{COMPLIANCE WITH ETHICS GUIDELINES CONFLICT OF INTEREST}

Annika Ohlsson, Mary Hunt, Anna Wedell and Ulrika von Döbeln declare that they have no conflict of interest.

\section{INFORMED CONSENT}

All procedures followed were in accordance with the ethical standards of the responsible committee on human experimentation (institutional and national) and with the Helsinki Declaration of 1975, as revised in 2000.

According to the ethical permission, informed consent was not requested.

Ethical permission: Genetic investigation of inborn errors of metabolism, approved by Regional Ethical Committee of Stockholm, 2008/351-31.

\section{CONTRIBUTION OF INDIVIDUAL AUTHORS}

Annika Ohlsson planned, designed and interpreted the data and wrote the draft of the manuscript.

Ulrika von Döbeln planned, designed and interpreted the data and revised the manuscript.

Mary Hunt planned and designed some of the experiments and revised the manuscript.
Anna Wedell interpreted data and revised the manuscript. All authors agree to submission of the manuscript.

Details of funding: Stockholm County Council and the Karolinska Institute Research Foundation.

\section{ORCID}

Annika Ohlsson (10 https://orcid.org/0000-0002-4115-1775

\section{REFERENCES}

1. Wada Y, Kikuchi A, Aria-Ichinoi N, et al. Biallelic GALM pathogenic variants cause a novel type of galactosemia. Genet Med. 2019; 21(6):1286-1294.

2. Kalckar HM, Anderson EP, Isselbacher KJ. Galactosemia, a congenital defect in a nucleotide transferase. Biochim Biophys Acta. 1956;20:262-268.

3. Rubio-Gozalbo ME, Haskowic M, Bosch AM, et al. The natural history of classic galactosemia: lessons from the GalNet registry. Orphanet J Rare Dis. 2019;14(1):86.

4. Waggoner D, Buist $\mathrm{N}$, Donnell G. Long-term prognosis in galactosaemia: results of a survey of 350 cases. J Inherit Metab Dis. 1990;13(6):802-818.

5. Carney AE, Sanders RD, Garza KR, et al. Origins, distribution and expression of Duarte-2 (D2) allele of galactose-1-phosphate uridyltransferase. Hum Mol Genet. 2009;18(9):1624-1632.

6. Levy HL, Sepe SJ, Walton DS, et al. Galactose-1-phosphate uridyltransferase deficiency due to Duarte/galactosemia combined variation: clinical and biochemical studies. J Pediatr. 1978;92(3): 390-393.

7. Carlock G, Fischer ST, Lynch ME, et al. Developmental outcomes in Duarte galactosemia. Pediatr. 2019;143(1):e20182516.

8. Ficicioglu C, Hussa C, Gallagher PR, Thomas N, Yager C. Monitoring of biochemical status of children with Duarte galactosemia: utility of galactose, galactitol, galactonate, and galactose-1-phosphate. Clin Chem. 2010;56(7):1177-1182.

9. Powell KK, Braun KVN, Singh RH, Shapira SK, Olney RS, Yeargin-Allsopp M. Long-term speech and language developmental issues among children with Duarte galactosemia. Genet Med. 2009;11(12):874-879.

10. Guthrie R, Susi A. A simple phenylalanine method for detecting phenylketonuria in large populations of newborn infants. Pediatrics. 1963;32(3):338-343.

11. Beutler E, Baluda MC. A simple spot screening test for galactosemia. J Lab Clin Med. 1966;68(1):137-141.

12. Loeber JG. Neonatal screening in Europe; the situation in 2004. J Inherit Metab Dis. 2007;30(4):430-438.

13. Alm J, Larsson A. Evaluation of a nation-wide neonatal metabolic screening programme in Sweden 1965-1979. Acta Paediatr Scand. 1981;70(5):601-607.

14. Ohlsson A, Guthenberg C, von Döbeln U. Galactosemia screening with low false-positive recall rate: the Swedish experience. JIMD Rep. 2012;2:113-117.

15. Reichardt J, Packman S, Woo S. Molecular characterization of two galactosemia mutations: correlation of mutations with highly conserved domains in galactose-1-phosphate uridyltransferase. Am J Hum Genet. 1991;49(4):860. 
16. Reichardt J, Woo S. Molecular basis of galactosemia: mutations and polymorphisms in the gene encoding human galactose1-phosphate uridyltransferase. Proc Natl Acad Sci U S A. 1991;88 (7):2633-2637.

17. Tyfield LA. Galactosaemia and allelic variation at the galactose1-phosphate uridyltransferase gene: a complex relationship between genotype and phenotype. Eur J Pediatr. 2000;159(Suppl 3):S204-S207.

18. Lai K, Langled SD, Singh RH, Dembure PP, Hjelm LN, Elsas LJ. A prevalent mutation for galactosemia among black Americans. J Pediatr. 1996;128(1):89-95.

19. Calderon FR, Phansalkar AR, Crockett DK, Miller M, Mao R. Mutation database for the galactose-1-phosphate uridyltransferase (GALT) gene. Hum Mutat. 2007;28(10):939-943.

20. Wadelius C, Lagerkvist A, Molin AK, Larsson A, von Döbeln U, Pettersson U. Galactosemia caused by a point mutation that activates cryptic donor splice site in the galactose-1-phosphate uridyltransferase gene. Genomics. 1993;17(2):525-526.

21. Den Dunnen J, Dalgleish R, Maglott DR, et al. HGVS recommendations for the description of sequence variants: 2016. Update Hum Mutat. 2016;37(6):564-569.

22. Adzhubei IA, Schmidt S, Peshkin L, et al. A method and server for predicting damaging missense mutations. Nat Methods. 2010;7 (4):248-249.

23. Choi Y, Sims GE, Murphy S, Miller JR, Chan AP. Predicting the functional effect of amino acid substitutions and indels. PLoS ONE. 2012;7(10):e46688.

24. Reese MG, Eeckman FH, Kulp D, Haussler D. Improved splice site detection in genie. J Comput Biol. 1997;4(3):311-323.

25. Orfanos AP, Jinks DC, Guthrie R. Microassay for estimation of galactose and galactose-1-phospate in dried blood specimens. Clin Biochem. 1986;19(4):225-228.

26. Allen JT, Holton JB, Gillett MG. Gas-liquid chromatographic determination of galactitol in amniotic fluid for possible use in prenatal diagnosis of galactosaemia. Clin Chim Acta. 1981;110(1): 59-63.

27. Shin-Bühring Y, Osang M, Ziegler R, Schaub J. A method for galactose-1-phosphate uridyltransferase assay and the separation of its isozymes by DEAE-cellulose column chromatography. Clin Chim Acta. 1976;70(3):371-377.

28. Boutron A, Marabotti A, Facchiano A, et al. Mutation spectrum in the French cohort of galactosemic patients and structural simulation of 27 novel missense variations. Mol Genet Metab. 2012;107 (3):438-447.

29. Bosch AM, Ijlst L, Oostheim W, et al. Identification of novel mutations in classical galactosemia. Hum Mutat. 2005;25(5):502.

30. Elsas LJ, Langley S, Steele E, et al. Galactosemia: a strategy to identify new biochemical phenotypes and molecular genotypes. Am J Hum Genet. 1995;56(3):630-639.

31. Tyfield L, Reichardt J, Fridovich-Keil J, et al. Classical galactosemia and mutations at the galactose-1-phosphate uridyltransferase (GALT) gene. Hum Mutat. 1999;13(6):417-430.
32. Leslie ND, Immerman EB, Flach JE, Florez M, Fridovich-Keil JÖ, Elsas LJ. The human galactose-1-phosphate uridyltransferase gene. Genomics. 1992;14(2):474-480.

33. Elsas LJ II, Langley S, Paulk EM, Hjelm LN, Dembure PP. A molecular approach to galactosemia. Eur J Pediatr. 1995;154(2): S21-S27.

34. Greber-Platzer S, Guldberg P, Scheibenreiter S, et al. Molecular heterogeneity of classical and Duarte galactosemia: mutation analysis by denaturing gradient gel electrophoresis. Hum Mutat. 1997; 10(1):49-57.

35. Bosch AM. Classical galactosaemia revisited. J Inherit Metab Dis. 2006;29(4):516-525.

36. Viggiano E, Marabotti A, Burlina AP, et al. Clinical and molecular spectra in galactosemic patients from neonatal screening in northeastern Italy: structural and functional characterization of new variations in the galactose-1-phosphate uridyltransferase (GALT) gene. Gene. 2015;559(2):112-118.

37. Novelli G, Reichardt JK. Molecular basis of disorders of human galactose metabolism: past, present, and future. Mol Genet Metab. 2000;71(1-2):62-65.

38. Ramadza DP, Sarnavka V, Vukovic J, et al. Molecular basis and clinical presentation of classical galactosemia in a Croatian population. J Pediatr Endocrinol Metab. 2018;31(1):71-75.

39. Gathof B, Sommer M, Podskarbi T, et al. Characterization of two stop codon mutations in the galactose-1-phosphate uridyltransferase gene of three male galactosemic patients with severe clinical manifestation. Hum Genet. 1995;96(6):721-725.

40. Kozàk L, Francovà H, Fajkusovà L, et al. Mutation analysis of the GALT gene in Czech and Slovak galactosemia populations: identification of six novel mutations, including a stop codon mutation (X380R). Hum Mutat. 2000;15(2):206.

41. Podskarbi T, Kohlmetz T, Gathof B, et al. Molecular characterization of Duarte-1 and Duarte-2 variants of galactose-1-phosphate uridyltransferase. J Inherit Metab Dis. 1996;19(5):638-644.

42. Yang YP, Corley N, Garcia-Heras J. Molecular analysis in newborns from Texas affected with galactosemia. Hum Mutat. 2002; 19(1):82-83.

\section{SUPPORTING INFORMATION}

Additional supporting information may be found online in the Supporting Information section at the end of this article.

How to cite this article: Ohlsson A, Hunt M, Wedell A, von Döbeln U. Heterogeneity of diseasecausing variants in the Swedish galactosemia population: Identification of 16 novel GALT variants. J Inherit Metab Dis. 2019;42:1008-1018. https://doi. org/10.1002/jimd.12136 